\title{
CONSISTENCY OF THE LEAST SQUARES ESTIMATOR OF THE AMPLITUDES AND ANGULAR FREQUENCIES OF A SUM OF HARMONIC OSCILLATIONS IN MODELS WITH LONG-RANGE DEPENDENCE
}

UDC 519.21

\author{
A. V. IVANOV
}

AbStract. The strong consistency of least squares estimators of unknown amplitudes and angular frequencies of the sum of harmonic oscillations observed in a strongly dependent Gaussian stationary noise is proved in the paper.

1. Let a stochastic process

$$
X(t)=g\left(t, \theta^{o}\right)+\varepsilon(t), \quad t \in[0, T]
$$

be observed, where

$$
\begin{gathered}
g\left(t, \theta^{o}\right)=\sum_{k=1}^{N}\left(A_{k}^{o} \cos \varphi_{k}^{o} t+B_{k}^{o} \sin \varphi_{k}^{o} t\right), \\
\theta^{o}=\left(\theta_{1}^{o}, \theta_{2}^{o}, \theta_{3}^{o}, \ldots, \theta_{3 N-2}^{o}, \theta_{3 N-1}^{o}, \theta_{3 N}^{o}\right)=\left(A_{1}^{o}, B_{1}^{o}, \varphi_{1}^{o}, \ldots, A_{N}^{o}, B_{N}^{o}, \varphi_{N}^{o}\right),
\end{gathered}
$$

$\left(A_{k}^{o}\right)^{2}+\left(B_{k}^{o}\right)^{2}>0, k=1, \ldots, N$; here $\varepsilon(t), t \in \mathbb{R}^{1}$, is a stochastic process defined on a complete probability space $(\Omega, \Im, \mathrm{P})$ and satisfying the following condition.

A1. $\varepsilon(t), t \in \mathbb{R}^{1}$, is a real, measurable, mean square continuous, stationary, Gaussian, zero-mean stochastic process.

We also assume that at least one of the following two conditions holds.

A2. The correlation function of the process $\varepsilon(t), t \in \mathbb{R}^{1}$, is such that

$$
\mathrm{E} \varepsilon(t) \varepsilon(0)=B(t)=\frac{L(|t|)}{|t|^{\alpha}}, \quad 0<\alpha<1,
$$

where $L(t)$ is a function slowly varying at infinity and $B(0)=1$.

A3.

$$
B(t)=\frac{\cos \varkappa t}{\left(1+t^{2}\right)^{\alpha / 2}}, \quad 0<\alpha<1, \varkappa \in[0, \infty)
$$

The statistical estimation of unknown amplitudes and angular frequencies (3) of a sum of harmonic oscillations (2) observed in a random noise $\varepsilon(t)$ is a probabilistic setting of the problem of detection of hidden periodicities. Investigations of this problem as well as of its deterministic counterpart $(\varepsilon(t) \equiv 0)$ are initiated by Lagrange. Many applications of this problem in numerous scientific fields are also known (see [1]).

2000 Mathematics Subject Classification. Primary 62J02; Secondary 62J99.

Key words and phrases. The detection of hidden periodicities, least squares estimator, consistency, long range dependence. 
The literature devoted to this question is rather extensive. We mention only a few mathematical publications [2]-6], where the consistency and asymptotic normality are studied for various statistical estimators of unknown amplitudes and angular frequencies under different assumptions concerning the stationary random noise $\varepsilon(t)$ in the model of observation (1), (2) with $N \geq 1$. Both cases of discrete and continuous time are studied in those papers. Similar problems are solved in [7, 8, for some other statistical models generalizing (1), (2).

The strong consistency of the least squares estimator of the parameter $\theta^{\circ}$ is studied in the present paper under conditions A1 and A2 or A3. The correlation function (4) is widely used in statistical applications (see, for example, 9, 10]). Properties of stochastic processes with the correlation function (5) are studied in [11.

2. We arrange the frequencies $\varphi^{o}=\left(\varphi_{1}^{o}, \ldots, \varphi_{N}^{o}\right)$ in ascending order. In other words, we assume that the parametric set where we search an estimator of unknown angular frequencies is of the following form:

$$
\Phi(\underline{\varphi}, \bar{\varphi})=\left\{\varphi=\left(\varphi_{1}, \ldots, \varphi_{N}\right) \in \mathbb{R}^{N}: 0 \leq \underline{\varphi}<\varphi_{1}<\cdots<\varphi_{N}<\bar{\varphi}<+\infty\right\} .
$$

Let

$$
Q_{T}(\theta)=T^{-1} \int_{0}^{T}[X(t)-g(t, \theta)]^{2} d t .
$$

According to the standard definition, the least squares estimator of the parameter $\theta^{\circ}$ constructed from observations after the process $X(t), t \in[0, T]$, is any random vector

$$
\theta_{T}=\left(A_{1 T}, B_{1 T}, \varphi_{1 T}, \ldots, A_{N T}, B_{N T}, \varphi_{N T}\right)
$$

that minimizes the functional $Q_{T}(\theta)$ in the set of parameters $\Theta \subset \mathbb{R}^{3 N}$ (the amplitudes $A_{k}, B_{k}, k=1, \ldots, N$, can assume arbitrary values in $\Theta$ ) for $\varphi \in \Phi^{c}$, where $\Phi^{c}$ is the completion of the set $\Phi(\underline{\varphi}, \bar{\varphi})$.

When proving the consistency of the estimator $\theta_{T}$ (see the theorem below) we face the problem of studying the behavior as $T \rightarrow \infty$ of the ratios

$$
\frac{\sin T\left(\varphi_{k T}-\varphi_{j T}\right)}{T\left(\varphi_{k T}-\varphi_{j T}\right)}, \quad \frac{\sin T\left(\varphi_{k T}-\varphi_{j}^{o}\right)}{T\left(\varphi_{k T}-\varphi_{j}^{o}\right)}, \quad k \neq j, \quad \frac{\sin T \varphi_{k T}}{T \varphi_{k T}}, \quad k=1, \ldots, N .
$$

However, the above definition of the estimator $\varphi_{T}=\left(\varphi_{1 T}, \ldots, \varphi_{N T}\right)$ makes it impossible to determine the behavior of the differences $\varphi_{k T}-\varphi_{j T}$ and $\varphi_{k T}-\varphi_{j}^{o}, j \neq k$, as $T \rightarrow \infty$. Therefore the question on the behavior of ratios (9) remains open.

Walker [3] proposed a modification of the definition of the estimator $\varphi^{\circ}$ which guarantees the convergence to zero of the ratios (9). In its turn, this implies the consistency of the least squares estimator.

The Walker idea is to define the estimator (8) as a point of minimum of the functional (7) in a set where one can well separate the parameters $\varphi_{k}$.

Consider a nondecreasing family of open sets

$$
\Phi_{T} \subset \Phi(\underline{\varphi}, \bar{\varphi}), \quad T \geq T_{0}>0 .
$$

We assume that these sets contain the true value of the parameter $\varphi^{\circ}$ and satisfy the following conditions:

$$
\begin{gathered}
\lim _{T \rightarrow \infty} \inf _{\substack{1 \leq j<k \leq N \\
\varphi \in \Phi_{T}}} T\left(\varphi_{k T}-\varphi_{j T}\right)=+\infty \\
\quad \lim _{T \rightarrow \infty \varphi \in \Phi_{T}} \inf _{T} T \varphi_{1}=+\infty .
\end{gathered}
$$


In view of the above remark, we say that a vector $\theta_{T}$ is the least squares estimator if $\theta_{T}$ is a point of minimum of the functional $Q_{T}(\theta)$ in the set $\Theta_{T}$ for which (in contrast with $\Theta) \varphi \in \Phi_{T}^{c}$.

Condition (12) obviously holds if $\underline{\varphi}>0$. If $\Phi_{T} \subset \Phi(0, \bar{\varphi})$, then one can consider the parametric sets such that

$$
\inf _{1 \leq j<k \leq N, \varphi \in \Phi_{T}} T\left(\varphi_{k}-\varphi_{j}\right)=T^{-1 / 2}, \quad \inf _{\varphi \in \Phi_{T}} T \varphi_{1}=T^{-1 / 2}
$$

in order to satisfy (11), (12).

Theorem. Let assumption A1 hold. If either $\mathbf{A 2}$ with a nondecreasing function $L$ or A3 holds, then the least squares estimator $\theta_{T}$ is a strongly consistent estimator of the parameter $\theta^{\circ}$, namely:

$$
\begin{gathered}
A_{k T} \rightarrow A_{k}^{o}, \quad B_{k T} \rightarrow B_{k}^{o}, \quad T\left(\varphi_{k T}-\varphi_{k}^{o}\right) \rightarrow 0 \quad \text { a.s. } \\
\text { as } T \rightarrow \infty, \quad k=1, \ldots, N .
\end{gathered}
$$

3. First we prove two auxiliary results.

Lemma 1. If assumptions $\mathbf{A} 1$ and $\mathbf{A} 2$ with a nondecreasing slowly varying function $L(t)$, $t \geq 0$, hold, then

$$
\xi(T)=\sup _{\lambda \in \mathbb{R}^{1}} T^{-\rho}\left|\int_{0}^{T} e^{-i \lambda t} \varepsilon(t) d t\right| \underset{T \rightarrow \infty}{\longrightarrow} 0 \quad \text { a.s. }
$$

for $\rho>1-\alpha / 6$.

Proof. Note that

$$
\begin{aligned}
\left|\int_{0}^{T} e^{-i \lambda t} \varepsilon(t) d t\right|^{2} & =\int_{-T}^{T} e^{-i \lambda u} \int_{0}^{T-|u|} \varepsilon(t+|u|) \varepsilon(t) d t d u \\
& =2 \int_{0}^{T} \cos \lambda u \int_{0}^{T-u} \varepsilon(t+u) \varepsilon(t) d t d u .
\end{aligned}
$$

Therefore

$$
\mathrm{E} \xi^{2}(T) \leq \frac{2}{T^{2 \rho}} \int_{0}^{T} \mathrm{E}\left|\int_{0}^{T-u} \varepsilon(t+u) \varepsilon(t) d t\right| d u \leq \frac{2}{T^{2 \rho}} \int_{0}^{T} \Psi^{1 / 2}(u) d u,
$$

where

$$
\begin{aligned}
\Psi(u)= & \int_{0}^{T-u} \int_{0}^{T-u} \mathrm{E} \varepsilon(t+u) \varepsilon(s+u) \varepsilon(t) \varepsilon(s) d t d s \\
= & (T-u)^{2} B^{2}(u)+\int_{0}^{T-u} \int_{0}^{T-u} B^{2}(t-s) d t d s \\
& +\int_{0}^{T-u} \int_{0}^{T-u} B(t-s+u) B(t-s-u) d t d s \\
= & \Psi_{1}(u)+\Psi_{2}(u)+\Psi_{3}(u) .
\end{aligned}
$$

Since

$$
\Psi^{1 / 2}(u) \leq \Psi_{1}^{1 / 2}(u)+\Psi_{2}^{1 / 2}(u)+\Psi_{3}^{1 / 2}(u),
$$

we have

$$
\begin{aligned}
\mathrm{E} \xi^{2}(T) & \leq \frac{2}{T^{2 \rho}} \int_{0}^{T} \Psi_{1}^{1 / 2}(u) d u+\frac{2}{T^{2 \rho}} \int_{0}^{T} \Psi_{2}^{1 / 2}(u) d u+\frac{2}{T^{2 \rho}} \int_{0}^{T} \Psi_{3}^{1 / 2}(u) d u \\
& =I_{1}(T)+I_{2}(T)+I_{3}(T)
\end{aligned}
$$


Each term on the right hand side of (19) is estimated separately. Put

$$
b_{u}(t-s)=B(t-s+u) B(t-s-u) .
$$

Then we make the change of variables in the integral $\Psi_{3}(u)$, namely $t \rightarrow t / T, s \rightarrow S / T$, and finally $t-s \rightarrow t$ :

$$
\begin{aligned}
\Psi_{3}(u) & =T^{2} \int_{0}^{1-\frac{u}{T}} \int_{0}^{1-\frac{u}{T}} b_{u}(T(t-s)) d t d s \\
& =T^{2}\left(1-\frac{u}{T}\right) \int_{-1+\frac{u}{T}}^{1-\frac{u}{T}}\left(1-\frac{|t|}{1-\frac{u}{T}}\right) b_{u}(T t) d t \leq T^{2}\left(1-\frac{u}{T}\right) \int_{-1}^{1} b_{u}(T t) d t \\
& \leq T^{2}\left(1-\frac{u}{T}\right)\left[\int_{0}^{1} B(T t+u) d t+\int_{-1}^{0} B(T t-u) d t\right]
\end{aligned}
$$

Since the correlation function $B$ is even,

$$
\int_{-1}^{0} B(T t-u) d t=\int_{0}^{1} B(T t+u) d t
$$

and hence

$$
\Psi_{3}(u) \leq 2 T^{2}\left(1-\frac{u}{T}\right) \int_{0}^{1} B(T t+u) d t .
$$

The monotonicity of the slowly varying function $L$ involved in condition $\mathbf{A 2}$ implies that

$$
B(T t+u)=\frac{L(T t+u)}{(T t+u)^{\alpha}} \leq \frac{L(2 T)}{(T t)^{\alpha}} \leq \frac{1+\varepsilon}{t^{\alpha}} \cdot \frac{L(T)}{T^{\alpha}}=\frac{1+\varepsilon}{t^{\alpha}} B(T)
$$

for all $\varepsilon>0$ and for all sufficiently large $T$ (say, for $T>T_{0}$ ). Thus

$$
\Psi_{3}(u) \leq \frac{2(1+\varepsilon)}{1-\alpha} L(T) T^{2-\alpha}\left(1-\frac{u}{T}\right)
$$

and

$$
I_{3}(T) \leq \frac{4 \sqrt{2}}{3}\left(\frac{1+\varepsilon}{1-\alpha}\right)^{1 / 2} L^{1 / 2}(T) T^{2-2 \rho-\alpha / 2} .
$$

The right hand side of (24) converges to zero as $T \rightarrow \infty$ if

$$
2-\frac{\alpha}{2}-2 \rho<0 \quad \text { or } \quad \rho>1-\frac{\alpha}{4} .
$$

The latter inequality follows from the assumptions of the lemma.

Similar reasoning proves a bound for $\Psi_{2}(u)$. This bound is, in fact, a particular case of inequality (21) corresponding to $u=0$ :

$$
\Psi_{2}(u) \leq 2 T^{2}\left(1-\frac{u}{T}\right) \int_{0}^{1} B^{2}(T t) d t \leq 2 T^{2}\left(1-\frac{u}{T}\right) \int_{0}^{1} B(T t) d t .
$$

Therefore

$$
I_{2}(T) \leq \frac{4 \sqrt{2}}{3(1-\alpha)^{1 / 2}} L^{1 / 2}(T) T^{2-2 \rho-\alpha / 2} .
$$

On the other hand,

$$
\Psi_{1}^{1 / 2}(u)=(T-u) B(u)
$$


whence

$$
\begin{aligned}
I_{1}(T) & =\frac{2}{T^{2 \rho}} \int_{0}^{T}(T-u) B(u) d u=2 T^{2-2 \rho} \int_{0}^{1}(1-u) B(T u) d u \\
& \leq 2 T^{2-2 \rho} \int_{0}^{1} B(T u) d u \leq \frac{2}{1-\alpha} T^{2-2 \rho-\alpha} L(T) .
\end{aligned}
$$

The right hand side of (27) converges to zero as $T \rightarrow \infty$ if

$$
2-2 \rho-\alpha<0 \quad \text { or } \quad \rho>1-\frac{\alpha}{2} \text {. }
$$

Thus

$$
\mathrm{E} \xi^{2}(T)=O\left(L^{1 / 2}(T) T^{2-2 \rho-\alpha / 2}\right)
$$

as $T \rightarrow \infty$.

Let $T_{n}=n^{\beta}$, where the number $\beta>0$ is such that

$$
\left(2 \rho+\frac{\alpha}{2}-2\right) \beta=1+\Delta, \quad \Delta>0 .
$$

Then $\sum_{n=1}^{\infty} \mathrm{E} \xi^{2}\left(T_{n}\right)<+\infty$; that is,

$$
\xi\left(T_{n}\right) \underset{n \rightarrow \infty}{\longrightarrow} 0 \text { almost surely. }
$$

Consider a sequence of random variables

$$
\begin{aligned}
\zeta_{n} & =\sup _{T_{n} \leq T<T_{n+1}}\left|\xi(T)-\xi\left(T_{n}\right)\right| \\
& =\sup _{T_{n} \leq T<T_{n+1}}\left|\sup _{\lambda \in \mathbb{R}^{1}}\right| \frac{1}{T^{\rho}} \int_{0}^{T} e^{-i \lambda t} \varepsilon(t) d t\left|-\sup _{\lambda \in \mathbb{R}^{1}}\right| \frac{1}{T_{n}^{\rho}} \int_{0}^{T_{n}} e^{-i \lambda t} \varepsilon(t) d t|| \\
& \leq \sup _{T_{n} \leq T<T_{n+1}} \sup _{\lambda \in \mathbb{R}^{1}}\left|\frac{1}{T^{\rho}} \int_{0}^{T} e^{-i \lambda t} \varepsilon(t) d t-\frac{1}{T_{n}^{\rho}} \int_{0}^{T_{n}} e^{-i \lambda t} \varepsilon(t) d t\right| \\
& \leq \sup _{T_{n} \leq T<T_{n+1}}\left[\sup _{\lambda \in \mathbb{R}^{1}}\left|\left(\frac{1}{T^{\rho}}-\frac{1}{T_{n}^{\rho}}\right) \int_{0}^{T_{n}} e^{-i \lambda t} \varepsilon(t) d t\right|\right. \\
& \left.\quad+\sup _{\lambda \in \mathbb{R}^{1}}\left|\frac{1}{T^{\rho}} \int_{T_{n}}^{T} e^{-i \lambda t} \varepsilon(t) d t\right|\right] \\
& \leq \frac{T_{n+1}^{\rho}-T_{n}^{\rho}}{T_{n}^{\rho}} \xi\left(T_{n}\right)+\frac{1}{T_{n}^{\rho}} \int_{T_{n}}^{T_{n+1}}|\varepsilon(t)| d t=\zeta_{n}^{(1)}+\zeta_{n}^{(2)} .
\end{aligned}
$$

It is clear that

$$
\zeta_{n}^{(1)} \underset{n \rightarrow \infty}{\longrightarrow} 0 \text { almost surely. }
$$

Consider

$$
\mathrm{E}\left(\zeta_{n}^{(2)}\right)^{2 k}=\frac{1}{T_{n}^{2 k \rho}} \int_{T_{n}}^{T_{n+1}} \cdots \int_{T_{n}}^{T_{n+1}} \mathrm{E} \prod_{j=1}^{2 k}\left|\varepsilon\left(t_{j}\right)\right| d t_{1} \cdots d t_{2 k}
$$

Since

$$
\mathrm{E} \prod_{j=1}^{2 k}\left|\varepsilon\left(t_{j}\right)\right| \leq \mathrm{E} \varepsilon^{2 k}(0)=(2 k-1) ! !
$$


we have

$$
\begin{aligned}
\mathrm{E}\left(\zeta_{n}^{(2)}\right)^{2 k} & \leq(2 k-1) ! ! \frac{\left(T_{n+1}-T_{n}\right)^{2 k}}{T_{n}^{2 k \rho}} \\
& =(2 k-1) ! !\left[\left(\frac{T_{n+1}}{T_{n}}-1\right) T_{n}^{1-\rho}\right]^{2 k}=O\left(n^{-2 k(1+\rho \beta-\beta)}\right) .
\end{aligned}
$$

If $1+\rho \beta-\beta>0$, then the series $\sum_{n=1}^{\infty} \mathrm{E}\left(\zeta_{n}^{(2)}\right)^{2 k}$ converges if $k$ is chosen appropriately. In its turn, the convergence of the latter series implies that

$$
\zeta_{n}^{(2)} \underset{n \rightarrow \infty}{\longrightarrow} 0 \text { almost surely. }
$$

Using equality (29), we obtain

$$
1+\rho \beta-\beta=1+\frac{(\rho-1)(1+\Delta)}{2 \rho+\frac{\alpha}{2}-2}>0
$$

or

$$
\rho>1-\frac{\alpha}{2(3+\Delta)} .
$$

By assumption, $\rho>1-\alpha / 6$. If $\rho$ is fixed, then one can choose $\Delta>0$ such that inequality (32) holds. Note that inequality (32) obviously holds if $\rho \geq 1$. Let

$$
1-\alpha / 6<\rho<1 \text {. }
$$

Then, for example,

where

$$
\rho>1-\frac{\alpha}{6}+\frac{\rho-\left(1-\frac{\alpha}{6}\right)}{2}=1-\frac{\alpha}{2+3 \Delta},
$$

$$
\Delta=\frac{3\left(\frac{\alpha}{6}-1+\rho\right)}{1-\rho+\frac{\alpha}{6}}>0 .
$$

Therefore

$$
\zeta_{n} \underset{n \rightarrow \infty}{\longrightarrow} 0 \quad \text { almost surely }
$$

and Lemma 1 is proved.

Lemma 2. Assume conditions $\mathbf{A} 1$ and $\mathbf{A 3}$. Then the asymptotic relation (15) holds for $\rho>1-\alpha / 6$.

Proof. Similarly to (21)-(24) we obtain

$$
\Psi_{3}(u) \leq 2 T^{2}\left(1-\frac{u}{T}\right) \int_{0}^{1}|B(T t+u)| d t \leq \frac{2}{1-\alpha} T^{2-\alpha}\left(1-\frac{u}{T}\right)
$$

and

$$
I_{3}(T)=\frac{2}{T^{2 \rho}} \int_{0}^{T}\left|\Psi_{3}(u)\right|^{1 / 2} d u \leq \frac{4 \sqrt{2}}{3 \sqrt{1-\alpha}} T^{2-2 \rho-\alpha / 2} .
$$

The same bound holds for $I_{2}(T)$, too. Further,

$$
I_{1}(T)=2 T^{2-2 \rho} \int_{0}^{1}(1-u)|B(T u)| d u \leq \frac{2}{1-\alpha} T^{2-2 \rho-\alpha} .
$$

Then

$$
\mathrm{E} \xi^{2}(T)=O\left(T^{2-2 \rho-\alpha / 2}\right)
$$

as $T \rightarrow \infty$. Further reasoning is the same as that in the proof of Lemma 1 
4. The proof of the theorem uses the ideas of the paper [5].

Let

$$
x_{k T}=\frac{\sin T\left(\varphi_{k T}-\varphi_{k}^{o}\right)}{T\left(\varphi_{k T}-\varphi_{k}^{o}\right)}, \quad y_{k T}=\frac{1-\cos T\left(\varphi_{k T}-\varphi_{k}^{o}\right)}{T\left(\varphi_{k T}-\varphi_{k}^{o}\right)} .
$$

We show that

$$
A_{k T}=A_{k}^{o} x_{k T}-B_{k}^{o} y_{k T}+o(1), \quad B_{k T}=A_{k}^{o} y_{k T}+B_{k}^{o} x_{k T}+o(1)
$$

for $k=1, \ldots, N$, where $o(1)$ denotes, generally speaking, different stochastic processes approaching zero almost surely as $T \rightarrow \infty$.

Differentiating the functional $Q_{T}(\theta)$ with respect to the variables $A_{1}, \ldots, A_{N}$ and $B_{1}, \ldots, B_{N}$ we obtain the following system of linear equations for the least squares estimators $A_{k T}$ and $B_{k T}, k=1, \ldots, N$ :

$$
\begin{cases}\sum_{k=1}^{N} a_{k j}^{(1)}(T) A_{k T}+\sum_{k=1}^{N} b_{k j}^{(1)}(T) B_{k T}=c_{j}^{(1)}(T), & j=1, \ldots, N, \\ \sum_{k=1}^{N} a_{k j}^{(2)}(T) A_{k T}+\sum_{k=1}^{N} b_{k j}^{(2)}(T) B_{k T}=c_{j}^{(2)}(T), & j=1, \ldots, N,\end{cases}
$$

where we used the notation

$$
\begin{gathered}
\langle u(t), v(t)\rangle=\frac{1}{T} \int_{0}^{T} u(t) v(t) d t, \\
a_{k j}^{(1)}(T)=\left\langle\cos \varphi_{k T} t, \cos \varphi_{j T} t\right\rangle, \quad a_{k j}^{(2)}(T)=\left\langle\cos \varphi_{k T} t, \sin \varphi_{j T} t\right\rangle, \\
b_{k j}^{(1)}(T)=\left\langle\sin \varphi_{k T} t, \cos \varphi_{j T} t\right\rangle, \quad b_{k j}^{(2)}(T)=\left\langle\sin \varphi_{k T} t, \sin \varphi_{j T} t\right\rangle, \\
c_{j}^{(1)}(T)=\left\langle X(t), \cos \varphi_{j T} t\right\rangle, \quad c_{j}^{(2)}(T)=\left\langle X(t), \sin \varphi_{j T} t\right\rangle, \\
k, j=1, \ldots, N .
\end{gathered}
$$

Considering the properties (11) and (12) of the parametric set $\Phi_{T}$ (whose completion contains the value of the least squares estimator $\left.\varphi_{T}=\left(\varphi_{1 T}, \ldots, \varphi_{N T}\right)\right)$ we derive the following relations:

$$
\begin{gathered}
a_{k j}^{(1)}(T)=o(1), \quad k \neq j, \\
a_{k k}^{(1)}(T)=\frac{1}{2}+o(1), \quad a_{k j}^{(2)}(T)=o(1), \quad k, j=1, \ldots, N,
\end{gathered}
$$

and

$$
\begin{gathered}
b_{k j}^{(1)}(T)=a_{j k}^{(2)}(T)=o(1), \\
b_{k j}^{(2)}(T)=o(1), \quad k \neq j, \quad b_{k k}^{(2)}(T)=\frac{1}{2}+o(1), \quad k, j=1, \ldots, N .
\end{gathered}
$$

Further,

$$
c_{j}^{(1)}(T)=\left\langle\varepsilon(t), \cos \varphi_{j T} t\right\rangle+\left\langle g\left(t, \theta^{o}\right), \cos \varphi_{j T} t\right\rangle=d_{j}^{(1)}(T)+d_{j}^{(2)}(T),
$$

and moreover, $d_{j}^{(1)}(T)=o(1)$ by Lemmas 1 and 2 . Then

$$
\begin{aligned}
d_{j}^{(2)}(T) & =A_{j}^{o}\left\langle\cos \varphi_{j}^{o} t, \cos \varphi_{j T} t\right\rangle+B_{j}^{o}\left\langle\sin \varphi_{j}^{o} t, \cos \varphi_{j T} t\right\rangle+o(1) \\
& =\frac{1}{2}\left[A_{j}^{o} x_{j T}-B_{j}^{o} y_{j T}\right]+o(1), \quad j=1, \ldots, N .
\end{aligned}
$$

Analogously

$$
c_{j}^{(2)}(T)=\frac{1}{2}\left[A_{j}^{o} y_{j T}+B_{j}^{o} x_{j T}\right]+o(1), \quad j=1, \ldots, N .
$$

Now relations (38) follow from (39)-(43). 
Since $\left|x_{k T}\right|,\left|y_{k T}\right| \leq 1$, relations (38) imply that

$$
\left|A_{k T}\right|,\left|B_{k T}\right| \leq\left|A_{k}^{o}\right|+\left|B_{k}^{o}\right|+o(1), \quad k=1, \ldots, N .
$$

Let $\Delta g\left(t ; \theta_{1}, \theta_{2}\right)=g\left(t, \theta_{1}\right)-g\left(t, \theta_{2}\right)$ and $G_{T}\left(\theta_{1}, \theta_{2}\right)=\left\langle\Delta g\left(t ; \theta_{1}, \theta_{2}\right), \Delta g\left(t ; \theta_{1}, \theta_{2}\right)\right\rangle$. By the definition of the least squares estimator,

$$
Q_{T}\left(\theta_{T}\right) \leq Q_{T}\left(\theta^{\circ}\right)
$$

On the other hand,

$$
Q_{T}\left(\theta_{T}\right)-Q_{T}\left(\theta^{o}\right)=G_{T}\left(\theta_{T}, \theta^{o}\right)+2\left\langle\varepsilon(t), \Delta g\left(t ; \theta^{o}, \theta_{T}\right)\right\rangle,
$$

where

$$
\left\langle\varepsilon(t), \Delta g\left(t ; \theta^{o}, \theta_{T}\right)\right\rangle=o(1)
$$

in view of Lemmas 1 and 2 and bounds (44). Taking into account inequality (45), we obtain from (46) and (47) that

$$
G_{T}\left(\theta_{T}, \theta^{\circ}\right) \underset{T \rightarrow \infty}{\longrightarrow} 0 \text { almost surely. }
$$

Put

$$
g_{k T}(t)=A_{k T} \cos \varphi_{k T} t+B_{k T} \sin \varphi_{k T} t-A_{k}^{o} \cos \varphi_{k}^{o} t-B_{k}^{o} \sin \varphi_{k}^{o} t .
$$

Then

$$
G_{T}\left(\theta_{T}, \theta^{o}\right)=\sum_{k=1}^{N}\left\langle g_{k T}(t), g_{k T}(t)\right\rangle+2 \sum_{k<j}\left\langle g_{k T}(t), g_{j T}(t)\right\rangle .
$$

Using the above reasoning and bounds (44), we find that

$$
\begin{gathered}
\left\langle g_{k T}(t), g_{j T}(t)\right\rangle=o(1), \quad k \neq j, \\
\left\langle g_{k T}(t), g_{k T}(t)\right\rangle=\frac{1}{2}\left[A_{k T}^{2}+B_{k T}^{2}+\left(A_{k}^{o}\right)^{2}+\left(B_{k}^{o}\right)^{2}\right]-\left(A_{k T} A_{k}^{o}+B_{k T} B_{k}^{o}\right) x_{k T} \\
+\left(A_{k T} B_{k}^{o}-A_{k}^{o} B_{k T}\right) y_{k T}+o(1), \quad k=1, \ldots, N .
\end{gathered}
$$

Substituting relations (38) into (51) and considering (50), we deduce that

$$
\begin{aligned}
G_{T}\left(\theta_{T}, \theta^{o}\right) & =\frac{1}{2} \sum_{k=1}^{N}\left(\left(A_{k}^{o}\right)^{2}+\left(B_{k}^{o}\right)^{2}\right)\left(1-x_{k T}^{2}-y_{k T}^{2}\right)+o(1) \\
& =\frac{1}{2} \sum_{k=1}^{N}\left(\left(A_{k}^{o}\right)^{2}+\left(B_{k}^{o}\right)^{2}\right)\left(1-\left(\frac{\sin \frac{1}{2} T\left(\varphi_{k T}-\varphi_{k}^{o}\right)}{\frac{1}{2} T\left(\varphi_{k T}-\varphi_{k}^{o}\right)}\right)^{2}\right)+o(1) .
\end{aligned}
$$

Thus relation (48) holds if and only if

$$
T\left(\varphi_{k T}-\varphi_{k}^{o}\right) \underset{T \rightarrow \infty}{\longrightarrow} 0 \text { almost surely, } \quad k=1, \ldots, N .
$$

Relations (53) obviously imply that

$$
x_{k T} \underset{T \rightarrow \infty}{\longrightarrow} 1, \quad y_{k T} \underset{T \rightarrow \infty}{\longrightarrow} 0 \quad \text { almost surely, } \quad k=1, \ldots, N .
$$

The consistency of the estimators $A_{k T}$ and $B_{k T}$ follows from equalities (38). 
5. Note that

where

$$
\frac{\cos \varkappa t}{\left(1+t^{2}\right)^{\alpha / 2}}=\frac{L(|t|)}{|t|^{\alpha}} \cos \varkappa t
$$

$$
L(t)=\left(\frac{t^{2}}{1+t^{2}}\right)^{\alpha / 2}, \quad t \geq 0,
$$

is a nondecreasing slowly varying function. Thus one can use the following condition:

A4.

$$
B(t)=\frac{L(|t|)}{|t|^{\alpha}} \cos \varkappa t, \quad \alpha \in(0,1), \varkappa \in[0 ; \infty),
$$

instead of $\mathbf{A 2}$ and $\mathbf{A 3}$ where $L(t), t \geq 0$, is a nondecreasing slowly varying function and $B(0)=1$.

Corollary. Conditions A1 and A4 imply (14).

Proof. Indeed, an inspection of the proofs of Lemmas 1 and 2 shows that relation (15) holds for the correlation function of the form (55).

\section{BIBLIOGRAPHY}

1. M. G. Serebrennikov and A. A. Pervozvanskiŭ, The Detection of Latent Periodicities, Nauka, Moscow, 1965. (Russian)

2. P. Whittle, The simultaneous estimation of a time series harmonic components and covariance structure, Trabajos Estadistica 3 (1952), 43-57. MR0051487 (14:488a)

3. A. M. Walker, On the estimation of a harmonic component in a time series with stationary dependent residuals, Adv. Appl. Probab. 5 (1973), 217-241. MR0336943 (49:1716)

4. E. J. Hannan, The estimation of frequency, J. Appl. Probab. 10 (1973), 510-519. MR0370977 $(51: 7200)$

5. A. V. Ivanov, A solution of the problem of detecting hidden periodicities, Teor. Veroyatnost. i Mat. Statist. 20 (1979), 44-59; English transl. in Theor. Probab. Math. Statist. 20 (1980), 51-68. MR 529259 (80c:62107)

6. A. V. Ivanov, Asymptotic Theory of Nonlinear Regression, Kluwer Academic Publishers, Dordrecht, 1997. MR:1472234 (99h:62086)

7. P. S. Knopov, Optimal Estimators of Parameters of Stochastic Systems, Naukova Dumka, Kiev, 1981. (Russian) MR619692 (83d:62134)

8. A. Ya. Dorogovtsev, The Theory of Estimators of Random Processes, Vyshcha Shkola, Kiev, 1982. (Russian) MR668517 (84h:62122)

9. A. V. Ivanov and N. N. Leonenko, Statistical Analysis of Random Fields, Vyshcha Shkola, Kiev, 1986; English transl., Kluwer Academic Publishers, Dordrecht, 1989. MR917486 (89e:62125)

10. J. Beran, Statistics for Long-Memory Processes, Chapman and Hall, New York, 1994. MR.1304490 (96b:62138)

11. V. V. Anh, N. N. Leonenko, and V. P. Knopova, Continuous-time stochastic processes with cyclical long-range dependence, Aust. N. Z. J. Stat. 46 (2004), no. 2, 275-296. MR2076396 (2005e:60079)

Department of Mathematical Analysis and Probability Theory, National Technical University of Ukraine "KPI", Peremogy Avenue 37, Kiev 03056, Ukraine

E-mail address: ivanov@paligora.kiev.ua

Received 11/SEP/2008

Translated by OLEG KLESOV 